\title{
Multichannel Odor Sensor Utilizing Surface Plasmon Resonance
}

\author{
Hidehito Nanto*, Fumio Yagi, Hisanori Hasunuma, Yoshinori Takei, \\ Shin Koyama, Takeshi Oyabu ${ }^{1}$ and Takashi Mihara² \\ Advanced materials Science R\&D Center, Kanazawa Institute of Technology, \\ 3-1 Yatsukaho, Hakusan, Ishikawa 924-0838, Japan \\ ${ }^{1}$ Kanazawa Seiryo University, 10-1 Gosyo, Kanazawa, Ishikawa 920-8620, Japan \\ ${ }^{2}$ Future Creation lab., Olympus Corp., 2-3-1 Nishi-shinjuku, Shinjuku, Tokyo 163-0914, Japan
}

(Received January 27, 2009; accepted April 12, 2009)

Key words: $\quad$ odor sensor, surface plasmon resonance, molecular recognition membrane

A multichannel odor sensor based on surface plasmon resonance (SPR) is demonstrated. Each thin film of acrylic acid and $N, N$-dimethylacetamide as a molecular recognition membrane is deposited on a gold thin-film/glass substrate by plasma chemical vapor deposition (CVD). The sensor with an acrylic acid thin film as the molecular recognition membrane exhibits an excellent selectivity for ammonia gas and the sensor with an $N, N$-dimethylacetamide thin film exhibits an excellent selectivity for acetic acid gas. Two types of odor sensor that can simultaneously detect ammonia and acetic acid gases with excellent selectivities are prepared on one chip substrate, and their gas sensing characteristics are studied.

\section{Introduction}

Surface plasmon resonance (SPR) is an optical phenomenon in which incident light excites a charge-density wave at the interface between a highly conductive metal and a dielectric material. The conditions for excitation are determined by the permittivity of the metal and the dielectric material. SPR transduction is widely used as an analytical tool for measuring small changes in the refractive index of a thin region adjacent to a metal surface.

The optical excitation of surface plasmons on a thin metallic film has, therefore, been recognized as a promising technique for the sensitive detection of chemical species. ${ }^{(1)}$ Several methods have been employed to monitor the excitation of SPR by measuring the light reflected from a sensor interface. These include the analysis of angle modulation, ${ }^{(2)}$ wavelength modulation, ${ }^{(3)}$ intensity modulation ${ }^{(4)}$ and phase modulation. ${ }^{(5)}$ It may be possible to apply the SPR phenomenon to a highly sensitive odor sensor.

Odors, fragrances and aromas have been identified using arrays of gas sensors, such

${ }^{*}$ Corresponding author: e-mail: hnanto@neptune.kanazawa-it.ac.jp 
as quartz resonators or oxide semiconductors, in conjunction with associated pattern recognition analysis..$^{(6-13)}$ We have reported that a novel aroma-sensing method that uses the principal component analysis and neural-network pattern recognition of transient responses measured using synthetic-polymer-film-coated quartz resonator gas sensors is useful in distinguishing the aromas of various types of coffee, wine and alcohol. . $9,12,13)^{2}$ Recently, we have proposed that harmful gases such as ammonia, trimethylamine and triethylamine can be detected by measuring SPR using angle modulation. The technique of using a synthetic polymer film as a molecular recognition membrane is applied in an SPR sensor. ${ }^{(14)}$ The SPR odor sensor has several merits such as not being affected by electrical noise due to the use of light, possible integration of sensors on a chip and high sensitivity due to the change in refractive index owing to interaction between the molecular recognition membrane and adsorbed gases.

The objective of this study is to demonstrate a two-channel odor sensor with excellent selectivity for ammonia and acetic acid gases, which is based on the SPR technique with two different membranes for molecular recognition, and is prepared on one chip substrate by plasma chemical vapor deposition (CVD).

\section{Experimental Methods}

Optical SPR sensors are sensitive to changes in the refractive index of a sample near a sensor surface. The SPR was measured using the Kretschmann configuration (Toa DKK: PR-40), shown in Fig. 1, with a prism and a thin, highly conductive gold metal layer deposited on the prism base. An LED emitting $660 \mathrm{~nm}$ light was used as the light source to excite SPR. The SPR reflection spectrum (reflected light intensity versus angle of incidence with respect to the normal of the metal/dielectric interface) was measured by coupling transverse magnetically polarized monochromatic light into the prism and measuring the reflected light intensity of the ray exiting the prism versus the angle of incidence. The reflected light was measured using a CCD camera attached to

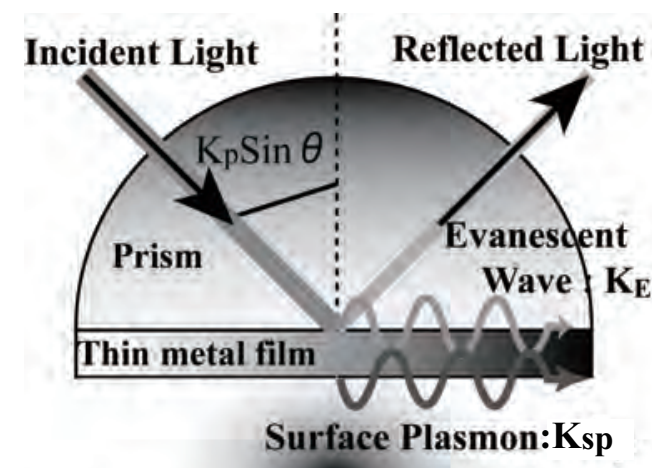

Sample

Fig. 1. Kretshmann configuration for generating the surface plasmon waves. 
a personal computer. The coupling of energy occurs between the incident light and the surface plasmon waves at the resonance angle, which gives the minimum intensity of the reflected light. The relationship ${ }^{(15)}$ between the resonance angle $\theta$ and the wave number of the surface plasmon $K_{\mathrm{SP}}$ is given by

$$
K_{\mathrm{P}} \sin \theta=K_{\mathrm{SP}}
$$

and

$$
K_{\mathrm{SP}}=\frac{\omega}{\mathrm{c}} \sqrt{\frac{\varepsilon n^{2}}{\varepsilon+n^{2}}},
$$

where $K_{\mathrm{P}}, K_{\mathrm{SP}}, \mathrm{c}, \varepsilon$ and $n$ are the wave number of the incident light, the angular frequency of the incident light, the speed of light in vacuum, the permittivity of the thin metal film and the refractive index of a sample near the sensor surface, respectively. Thus, an increase in resonance angle corresponds to an increase in the refractive index of a sample near the sensor surface in the SPR reflection spectrum. The schematic configuration of the SPR system is shown in Fig. 2. Four channel images (Channels 1-4) of reflected light can be observed using the CCD camera, because the CCD image can be divided into four channels using computer software.

To utilize this system as a novel odor sensor, each very thin film of acrylic acid and $N, N$-dimethylacetamide as the molecular recognition membrane was deposited on a gold $(\mathrm{Au})$ thin-film substrate by plasma CVD. A schematic of the plasma CVD system used is shown in Fig. 3. In this case, two channels of the CCD camera were used as the sensor, because two types of molecular recognition membranes were deposited on the $\mathrm{Au}$ substrate by plasma CVD. A vaporized monomer was introduced into the chamber evacuated to a base pressure of $10 \mathrm{~Pa}$ together with $\mathrm{Ar}$ as the carrier gas. The partial pressures of the monomer vapor and Ar gas were set to be approximately 20 and $80 \mathrm{~Pa}$,
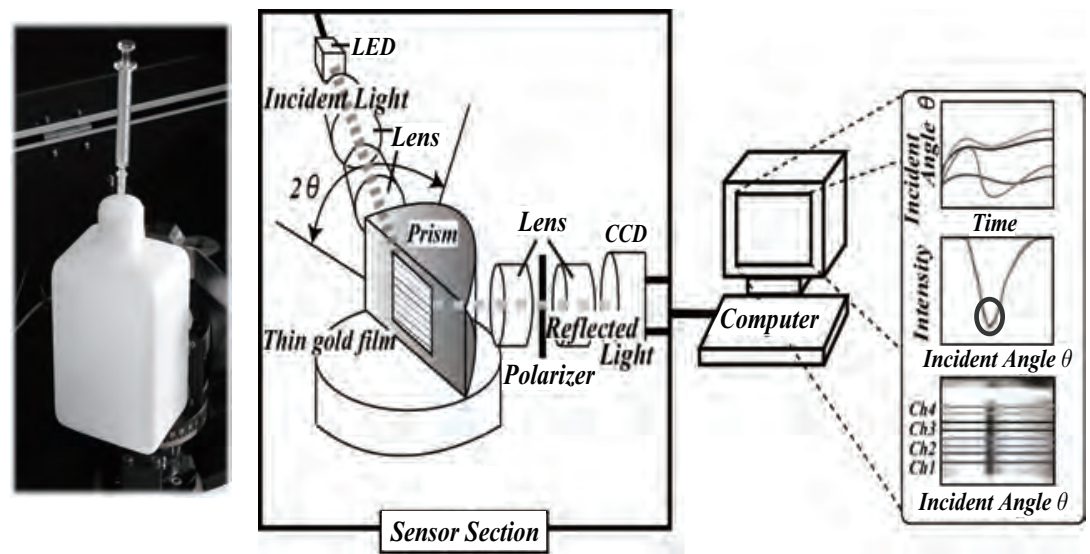

Fig. 2. Schematic diagram of SPR sensor system used. 


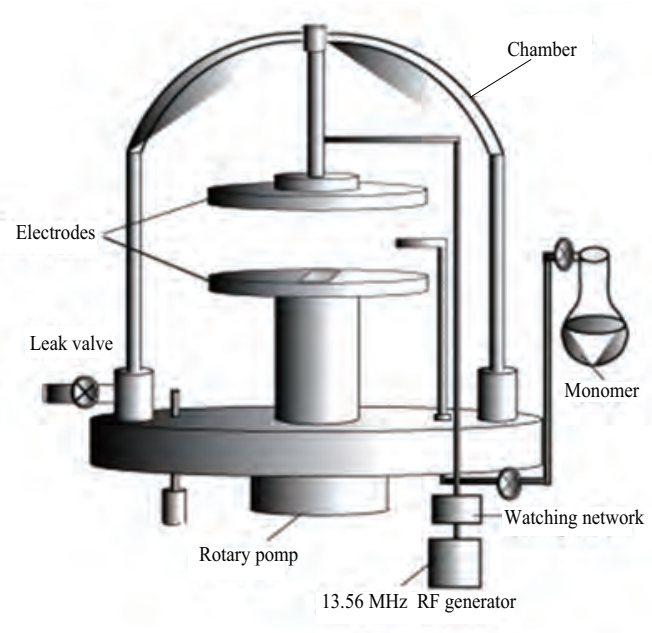

Fig. 3. Schematic of plasma CVD apparatus.

respectively. The Ar-based plasma was produced by the radio frequency (RF) discharge between two electrodes with a supplied power of $100 \mathrm{~W}$, and activated monomers polymerized on the surface of the Au thin film. Two types of monomer, namely acrylic acid with a carboxyl group and $N, N$-dimethylacetamide, were used as materials for the molecular recognition membrane. Thin films of both organic materials were deposited on the Au substrate, as shown in Fig. 4. The typical deposited thickness on the Au film was $50-80 \mathrm{~nm}$, which was monitored using a quartz resonator.

A test gas at concentrations of $50-400 \mathrm{ppm}$ was injected into the $500-\mathrm{ml}$ sensing cell at $20 \mathrm{~s}$ after the start of the measurement at a temperature of $20^{\circ} \mathrm{C}$ and a humidity of 55\%. The response of the SPR sensor was then monitored for $5 \mathrm{~min}$. Eleven harmful gases, namely ammonia, acetaldehyde, propionaldehyde, xylene, toluene, trimethylamine, triethylamine, dimethylamine, formaldehyde, acetic acid and butyl acetate, were used as test gases.

\section{Results and Discussion}

It was confirmed that each SPR odor sensor with the acrylic acid thin film (46 nm thick) and with the $N, N$-dimethylacetamide thin film (50 nm thick) as the molecular recognition membranes exhibited excellent selectivities for ammonia gas and acetic acid gas, respectively. Figure 5 shows a typical response of each SPR odor sensor with the acrylic acid and $N, N$-dimethylacetamide thin films as the molecular recognition membranes to various testing gases, indicating that the sensor did not respond to various testing gases other than ammonia and acetic acid gases. Figure 6 shows a typical CCD pattern of the SPR odor sensor. It can be seen that the SPR occurs at resonance angles of about $42^{\circ}$ and about $50^{\circ}$ (black part in the figure). This means that reflected light intensity is decreased at each resonant angle. 


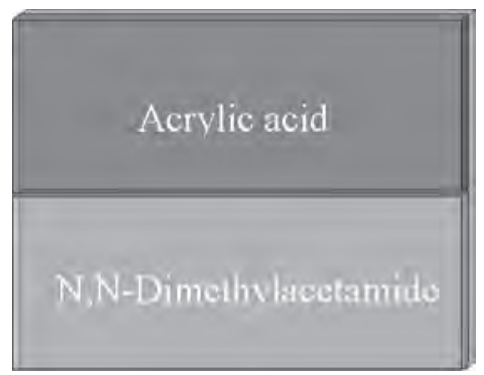

Fig. 4. Schematic of acrylic acid and dimethylacetoamide thin films deposited on Au thin film/ glass substrate $\left(25 \times 25 \mathrm{~mm}^{2}\right)$ by plasma CVD.

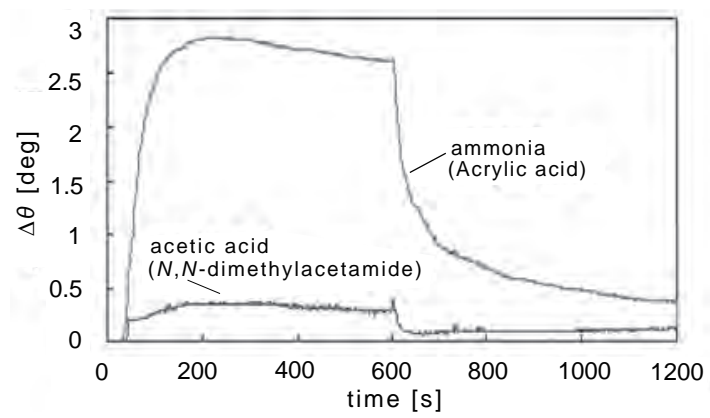

Fig. 5. Typical responses of SPR odor sensor coated with acrylic acid and $N, N$-dimethylacetoamide thin films to various gases.

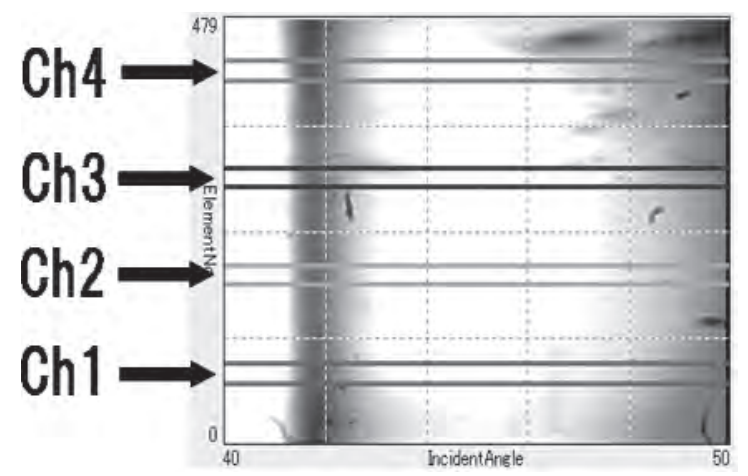

Fig. 6. CCD pattern of SPR odor sensor exposed simultaneously to ammonia and acetic acid gases at $t=0 \mathrm{~s}$.

Figure 7 shows a typical response of the SPR odor sensor with the acrylic acid and $N, N$-dimethylacetamide thin films to ammonia and acetic acid gases. Acetic acid gas was injected into the measuring chamber at time $t=0 \mathrm{~s}$ and the ammonia gas was injected into the chamber at time $t=300 \mathrm{~s}$. It can be seen that each sensor responded to acetic acid and ammonia gases with excellent selectivities. Figure 8 shows typical responses 


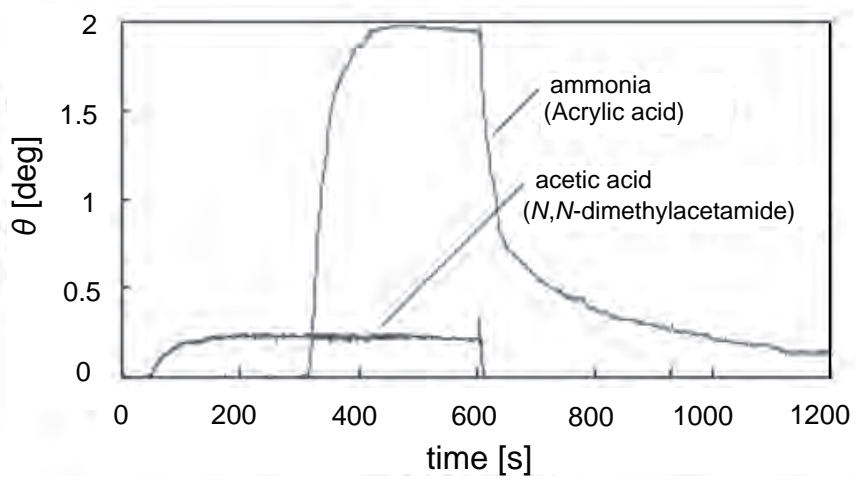

Fig. 7. Typical responses of SPR odor sensor coated with acrylic acid thin film and $N, N$ dimethylacetamide thin film. Acetic acid gas was injected into the measuring chamber at $t=0 \mathrm{~s}$ and then ammonia gas was injected into the chamber at $t=300 \mathrm{~s}$.

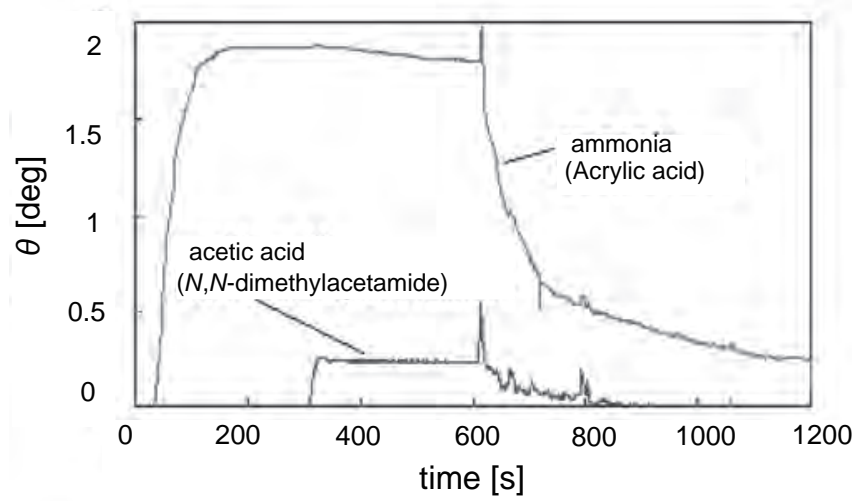

Fig. 8. Typical responses of SPR odor sensor coated with acrylic acid thin film and $N, N$ dimethylacetamide thin film. Ammonia gas was injected into the measuring chamber at $t=0 \mathrm{~s}$ and then acetic acid gas was injected into the chamber at $t=300 \mathrm{~s}$.

of the sensor when ammonia gas was injected into the chamber at $t=0 \mathrm{~s}$ and then acetic acid gas was injected into the chamber at $t=300 \mathrm{~s}$, indicating that in this case each sensor also responded to ammonia and acetic acid gases with very excellent selectivities, respectively.

It was confirmed that the output of each SPR odor sensor was increased with increasing gas concentration. Figure 9 shows typical responses of the SPR odor sensor with the acrylic acid molecular recognition film for ammonia gas with various concentrations, as an example. The rise time to reach a saturation value for the resonant angle change tended to decrease with increasing ammonia gas concentration. Figure 10 shows the saturation value of sensor output as a function of ammonia concentration. It can be seen that sensor output increases with increasing ammonia concentration. The 


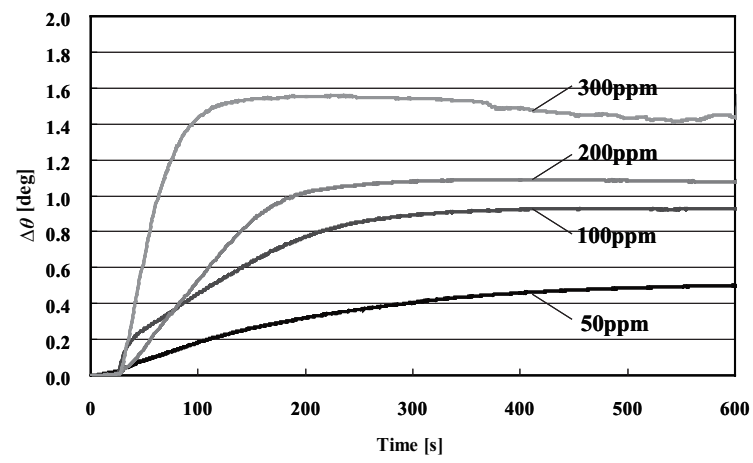

Fig. 9. Typical responses of SPR odor sensor coated with acrylic acid thin film for ammonia gas as a function of ammonia concentration.

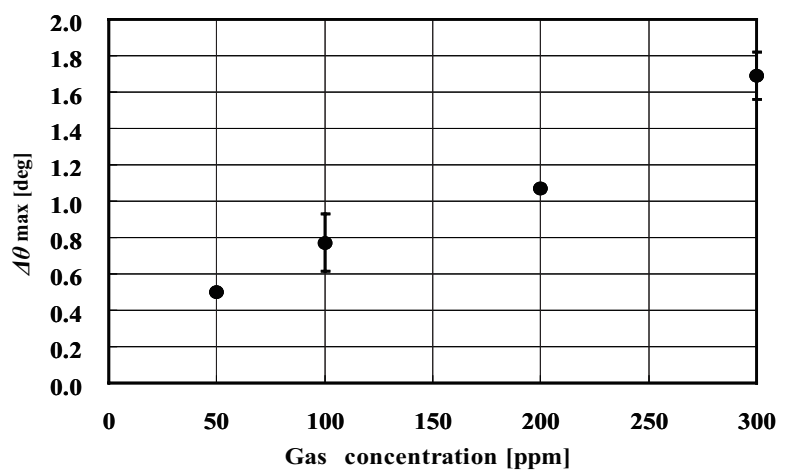

Fig. 10. Relationship between sensor output and ammonia gas concentration.

ammonia molecules adsorbed on the acrylic acid molecular recognition membrane surface may equilibrate with ammonia molecules in the gas phase at each equilibrium pressure for various concentrations of ammonia, which causes the coverage of ammonia molecules to be less than unity because the change in angle may saturate and become constant for various concentrations of ammonia for coverages less than unity. It was also confirmed that the output of the SPR odor sensor with the $N, N$-dimethylacetamide thin film as the molecular recognition membrane increased with increasing acetic acid gas concentration from 100 to $1,000 \mathrm{ppm}$. For the detection of ammonia, the lower limit of the sensitivity is about several ppm, as shown in Fig. 9, because the detection lower limit of resonant angle change is 0.1 .

The results described above strongly suggest that the SPR odor sensor deposited with the acrylic acid and $N, N$-dimethylacetamide membranes by plasma CVD is useful for detecting ammonia and acetic acid gases with excellent selectivity. It can be expected that a multichannel (4-channel) odor sensor can be prepared using an SPR sensor system and plasma CVD, because the SPR sensor system has four channels as analytical software, and four types of molecular recognition membrane can be deposited on one chip of a Au thin film/glass substrate by plasma CVD. 


\section{Summary}

An SPR odor sensor with an acrylic acid thin film and an $N, N$-dimethylacetamide thin film as the molecular recognition membrane was prepared on a Au thin-film/glass substrate by plasma CVD. The SPR odor sensor with an acrylic acid membrane exhibited excellent selectivity for ammonia gas. The SPR odor sensor with an $N, N$ dimethylacetamide membrane exhibited excellent selectivity for acetic acid gas. The output of the SPR odor sensor with both molecular recognition membranes deposited on a Au thin film increases with increasing concentration of each gas. We expect that the sensitivity of the SPR sensor can be improved by optimizing the thickness of the molecular recognition membrane. Right now, an experiment on the deposition of four types of molecular recognition membrane on one chip of the substrate is under way.

\section{Acknowledgements}

The authors wish to thank Prof. M. Habara of Kyushu University for his helpful discussion, and Y. Dehari, M. Takeda and N. Takebe for their excellent assistance with the experiments. This work was partially supported by the foundation for OpenResearch Center Program from the Ministry of Education, Culture, Sports, Science and Technology of Japan.

\section{References}

B. Liedberg, C. Nylander and I. Lundstrom: Sens. Actuators 4 (1983) 299.

E. Kretschmann: Z. Phys. 241 (1971) 313.

K. S. Johnston, S. R. Karlson, C. Jung and S.S. Yee: Mater. Chem. Phys. 42 (1995) 242.

B. Chadwick and M. Gal: Jpn. J. Appl. Phys. 32 (1993) 2716.

5 S. Nelson, K. S. Johnston and S. S. Yee: Sens. Actuators, B 35,36 (1998) 187.

6 W. P. Cary, K. E. R. Beebe and B. R. Kowalski: Anal. Chem. 58 (1989) 211.

7 T. Nakamoto, K. Fukunishi and T. Moriizumi: Sens. Actuators, B 1 (1990) 473.

8 R. Muller: Sens. Actuators, B 4 (1991) 35.

9 H. Nanto, T. Kawai, H. Sokooshi and T. Usuda: Sens. Actuators, B 13,14 (1992) 718.

10 T. Nakamoto, A. Fukuda and T. Moriizumi: Sens. Actuators, B 10 (1993) 85.

11 J. W. Gardner and P. N. Bartlet: Sens. Actuators, B 18,19 (1994) 211.

12 H. Nanto, K. Kondo, M. Habara, Y. Douguchi, R. I. Waite and H. Nakazumi: Sens. Actuators, B 35,36 (1996) 183.

13 H. Nanto, S. Tsubakino, M. Habara, K. Kondo, T. Morita, Y. Douguchi, H. Nakazumi and R. I. Waite: Sens. Actuators, B 34 (1996) 312.

14 H. Nanto, Y. Kitade, Y. Sekikawa, Y. Takei, N. Kubota, E. Kusano and A. Kinbara, Proc. SPIE $\mathbf{5 2 7 0}$ (2004) 174.

15 J. Homora, S. S. Yee and G. Gauglitz: Sens. Actuators, B 54 (1999) 3. 